\title{
REPRESENTACIONES SOBRE LA ESCRITURA EN El Discurso Docente EN El ConTEXTo DE LA ESCUELA SECUNDARIA. UNA INDAGACIÓN De Las Creencias Sobre Las Prácticas Y SU ORIENTACIÓN
}

$V$ anesa Condito

Universidad Nacional de Rosario

conditov@gmail.com

Resumen: Este artículo tiene como objetivo explorar las representaciones o configuraciones semiotizadas de conocimientos (Charaudeau, 2012, 2009, 1983; Bronckart, 2004, 2007) sostenidas por el discurso docente en torno de la escritura en la escuela secundaria. Se realizó un análisis cualitativo y cuantitativo de las declaraciones de 25 profesores de la ciudad de Rosario como respuesta a una encuesta con dos preguntas abiertas y una cerrada de opción múltiple. Se parte de la asunción de que en estas declaraciones y respuestas se movilizan representaciones que dan cuenta del plano imaginario (Bruner, 2009, 2004) en relación con la escritura en los contextos formativos, tanto respecto de las propias prácticas docentes como respecto de su orientación deóntica.

La principal tendencia identificada en el corpus reside en la representación de la escritura como dominio inespecífico y asociado con la funcionalidad escolar. Se considera que los resultados de este tipo de indagación permiten obtener indicadores válidos capaces de orientar eventuales intervenciones didácticas.

Palabras clave: Representaciones, Escritura, Docentes, Escuela secundaria

\begin{abstract}
This article aims to explore the representations or semiotic configurations of knowledge (Charaudeau, 2012, 2009, 1983; Bronckart, 2004, 2006) sustained by the educational discourse around writing in secondary school. A qualitative and quantitative analysis was made based on the statements of 25 teachers from Rosario, as a response to a survey including two questions with open answers and one closed multiple-choice question. We proceed under the assumption that these statements and answers mobilize representations that evidence an imaginary level (Bruner, 1990) related to writing in formative contexts, not only in the educational practices but also in its deontic orientation.

The main identified trend in the corpus lies on the representation of writing as a non-specific domain and associated with the school functionality. It is considered that the results of this type of inquiry allow obtaining valid indicators capable of guiding eventual didactic interventions.
\end{abstract}

Keywords: Representations, Writing, Teachers, Secondary School 


\section{Introducción}

Cuando pensamos en el objeto escritura y en las múltiples variables intervinientes en su definición, históricamente sometida a complejas caracterizaciones desde puntos de vista teóricos heterogéneos (Cardona, 1999; Olson, 1994; Olson y Torrance, 1998; Cavallo y Chartier, 2004; Catach, 1996), resulta evidente que, lejos de tratarse de un objeto de conocimiento "neutro" y "estable", estamos ante un terreno que -de manera semejante a lo que sucede con otros fenómenos culturales que se hallan en la base de la organización de nuestra sociedad- suscita más preguntas que respuestas dadas de antemano. ¿De qué hablamos cuando hablamos de escritura? ¿Qué significa leer y escribir en nuestra cultura? ¿Cuáles son los discursos, actores sociales e instituciones que intervienen en su definición?

Por otra parte, cuando a estos interrogantes le añadimos la pregunta por su modo de transmisión y adquisición, y, en particular, nos centramos en la significación que el objeto escritura asume en el marco de las prácticas educativas, el terreno deviene aún más complejo. ¿Qué significa leer y escribir en la escuela? ¿Existe algún tipo de consenso en el interior de las instituciones educativas respecto de lo que esta escritura "es" y/o "debería ser"?

Asimismo, más específicamente, si atendemos a la creciente proliferación de cursos, talleres, programas e incluso asignaturas curriculares surgidas en los últimos años destinadas al desarrollo de competencias dentro del dominio de la escritura en ingresantes a los estudios del nivel superior, resulta evidente que se manifiesta algún tipo de desajuste respecto de la manera en que se concibe (y transmite) la escritura en el marco de escuela secundaria y las necesidades propias de los trayectos posteriores de los estudiantes que de allí se egresan. En efecto, más allá de las divergencias en 
los enfoques y proyectos, estas propuestas de enseñanza parten de un diagnóstico en el que es posible hallar como "común denominador" la consideración de la escritura en términos de "problema", de modo tal que pareciera que leer y escribir, o devenir agente de lectura y escritura, se constituye como un obstáculo difícil de sortear en los contextos formativos en general, y en la escuela secundaria en particular (Cfr. Cárdenas, 2013; Delicia Martínez, 2016; Desinano y Solana, 2015; Falchini, 2006; Freytes y Fernández, 2016; Pérez y Rogieri, 2016; Riestra, 2010).

En virtud de esta problemática, y dado que entendemos a la escritura como un dominio de simbolización complejo y multidimensional -que involucra operaciones, sujetos y objetos(Condito, 2018, 2016, 2014) sometido a la determinación y variación histórico-cultural (Calvet, 2008; Cardona, 1999), consideramos legítimo y relevante preguntarnos acerca de sus sentidos y valores asociados. Más particularmente, si advertimos, además, que en nuestra sociedad se plantea un estrecho lazo históricamente fijado entre escritura y escuela (Chartier, 2005; Ferreiro, 2011; Petrucci, 1999) -en la medida en que la alfabetización deviene mandato educativo fundacional (Cucuzza y Pineau, 2012; Tedesco, 1993)- y, sobre todo, si asumimos que "todos los objetos sociales cuando pasan a ser escolares se transforman" (Ferreiro, 2011, p. 47), cabría interrogarnos por la manera en que este dominio es configurado específicamente en los espacios formativos institucionales.

Es decir, dado el planteo esbozado supra, surge como relevante indagar acerca de las representaciones (Bronckart, 2004, 2007; Charaudeau, 2009, 1983) sobre la escritura que se movilizan en la escuela, y en la escuela secundaria en particular.

Ahora bien, al centrarnos en el contexto de las instituciones escolares, es necesario señalar que, además de su diversidad inherente -en la medida en que toda institución escolar en el seno 
de una sociedad compleja y desigual manifiesta heterogeneidades (Bourdieu, 2015) -, en su interior se presentan distintos espacios discursivos pasibles de ser analizados. Esto es, a los fines de indagar las representaciones asociadas al dominio de la escritura en las instituciones educativas, es posible centrarse tanto en el plano prescriptivo (los diseños curriculares ministeriales, los planes de estudio, las planificaciones docentes, los proyectos institucionales), en el plano de las mediaciones formativas (las secuencias de interacciones en las clases, las consignas de trabajo, los materiales de estudio), o bien, en el plano más general de las creencias o imaginario (Bruner, 2009, 2004) de los agentes involucrados (docentes, alumnos, directivos, familias), que es el que aquí abordaremos.

En el presente artículo -que se enmarca en nuestra Tesis doctoral (Condito, 2018) orientada al estudio de las consignas en la escuela secundaria y las representaciones sobre la escritura que estas instituyen-, en efecto, tenemos como objetivo explorar y sistematizar las creencias sostenidas y movilizadas por el discurso docente en torno de la escritura. Más específicamente, abordaremos las declaraciones de 25 docentes de distintas asignaturas y escuelas secundarias de la ciudad de Rosario como respuesta a una breve encuesta con dos preguntas abiertas y una cerrada que elaboramos para tales fines, en la medida en que consideramos que estas declaraciones pueden ser concebidas como portadoras de representaciones o configuraciones semiotizadas de conocimientos (Charaudeau, 2012, 2009, 1983; Bronckart, 2004, 2007) que dan cuenta del plano "imaginario" (Bruner, 2009, 2004) en relación con la escritura en los contextos formativos.

Este instrumento de la encuesta, cuyos resultados presentaremos y discutiremos en el presente escrito -luego de una sucinta formulación de los lineamientos teóricos y metodológicos que sostuvieron nuestra investigación-, estuvo específicamente orientado a indagar las representaciones acerca de la escritura en 
el discurso docente, tanto en relación con "el ser" como respecto del "deber ser". Es decir, su objetivo fue explorar la manera en que los docentes conciben sus propias prácticas formativas relativas a la escritura y ponerlas en relación con las expectativas que manifiestan respecto de tales prácticas -su orientación deóntica-, a los fines de, posteriormente, establecer una confrontación entre ambas instancias y evaluar si se plantea o no coherencia entre ambos niveles para, de esta forma, obtener indicadores válidos capaces de orientar eventuales intervenciones didácticas.

\section{Lineamientos teóricos y metodológicos}

La investigación que enmarca este trabajo se inscribe en un doble campo disciplinar, esto es, pretende poner en relación el área de la didáctica de la lengua con el análisis del discurso en la medida en que establece una indagación analítica orientada a relevar determinadas constancias y dominancias interdiscursivas respecto de la escritura en tanto que objeto de enseñanza.

Retomamos la caracterización del campo de la didáctica de la lengua que propone Bronckart $(2004,2007)$ en tanto área disciplinar específica que organiza su dominio en la interrelación entre la Lingüística, la Psicología del desarrollo y las Ciencias de la Educación, y que tiene cuatro principales ejes de investigación: 1) el que enfoca el estado de la enseñanza de la lengua materna 2) el que aborda los procesos de transposición didáctica 3) el que contempla el análisis de las interacciones de enseñanza-aprendizaje que se desarrolla en las clases 4) el que se orienta de modo intervencionista en función de construir nuevos procedimientos de acción formativa. En particular, nuestra investigación se centra en el eje relativo a los procesos de transposición. Esto es así puesto que se orienta a comprender el complejo vínculo y transformaciones involucradas entre los objetos del saber -en este 
caso, el objeto escritura- y los objetos de enseñanza -la escritura en la escuela secundaria-, desde la premisa de que "los saberes son puestos en circulación, reproducidos, rechazados, transformados, en el marco de la actividad verbal humana" (Bronckart, 2007, p. 117).

En este sentido, asumimos también como punto de partida que el "decir" -siempre entendido performativamente (Bourdieu, 2001) - es parte constitutiva e integrante del "hacer", en virtud de que lo que se dice acerca de lo que se hace y acerca de lo que se debería hacer también conforma "la constitución social de lo real desde el plano de lo imaginario" (Bruner, 2009, p. 34), y de allí la relevancia que le adjudicamos a este tipo de análisis centrado en el discurso docente.

En cuanto al análisis del discurso, partimos de su definición como área interdisciplinar que tiene como objeto el estudio la relación entre lenguaje, sentido y vínculo social (Charaudeau, 1995) y, desde allí, se propone intentar percibir y sistematizar regularidades en lo pensable y decible en una coyuntura determinada (Angenot, 2010). Asimismo, y en consecuencia, se asume que el discurso:

no es solamente lo que se dice, pronuncia o escribe en su aspecto explícito, a través de un cierto modo verbal. Es un sistema de significación que se transporta por debajo de lo que se dice explícitamente; así el discurso es el resultado de una combinación entre lo explícito y lo implícito de lo que se dice, ya sea una conversación oral, un texto escrito o una imagen. (Charaudeau, 2012, p. 101).

En función de este posicionamiento teórico, en primer lugar, interesa señalar que inscribimos a nuestras unidades de análisis las respuestas-declaraciones de los docentes- en el contexto de lo que denominamos el "ámbito de la praxis y discursividad escolar", al que definimos como campo (Bourdieu, 2002, 2001) específico de producción de discursos y saberes que, en cuanto tal, involucra un sistema de posiciones de agentes, reglas y relaciones de 
producción que se articulan con determinados modos de decir y de validar lo dicho. Asimismo, resulta relevante aclarar -y, de este modo, establecer explícitamente el lazo entre análisis del discurso y didáctica de la lengua que proponemos- que una de las propiedades particulares de este campo es la de dar lugar a lo que Chevallard (1985) denomina "sistema didáctico", esto es, una formación tecnocultural que organiza una relación entre tres polos: el docente, el alumno y el objeto de saber.

Por otra parte, en virtud de que nuestra variable de indagación en las declaraciones-respuestas de los docentes a la encuesta son, como dijimos, las representaciones o mundos representados con pretensiones de validez designativa en torno de la escritura que allí se sostienen y movilizan, conceptualizamos las representaciones en términos de configuraciones verbales interdiscursivas acerca del objeto escritura, producidas en y por las actividades formativas en el contexto de las instituciones escolares

históricamente situadas y socialmente reguladas por un campoque operan como constitutivas y constituyentes de discursos y prácticas, y que en cuanto tales presentan necesariamente una tensión entre homogeneidad y dispersión (Angenot, 2010; Charaudeau, 2012).

En lo que concierne al plano metodológico, trabajamos a partir de un corpus constituido por 149 declaraciones-respuestas docentes producto de 25 encuestas. Para su obtención, se solicitó la colaboración de distintos profesores de Lengua y Literatura de distintas escuelas secundarias de la ciudad de Rosario a los fines de que distribuyeran la encuesta entre sus compañeros de otras asignaturas. Posteriormente, tales colaboradores nos remitieron vía correo electrónico las respuestas que pudieron recolectar. De esta manera se intentó garantizar no solo el anonimato sino también la heterogeneidad de la población: las encuestas recolectadas son respondidas por docentes que se desempeñan en 
los distintos años de escolaridad secundaria en un total de 17 escuelas, tanto públicas como privadas, orientadas y técnicas.

Cabe señalar que decidimos deliberadamente excluir a los docentes de Lengua y Literatura de la población encuestada a los fines de evitar sesgos en las respuestas en virtud de las posibles interferencias que habilitaría su consciencia metalingüística. Por otra parte, esta decisión se fundamenta en la medida en que consideramos que, lejos de ser "patrimonio" exclusivo de los docentes de Lengua, la escritura constituye un objeto de enseñanza que atraviesa todas las áreas disciplinarias en la escuela secundaria (Navarro y Revel Chion, 2013).

Presentamos, a continuación, la encuesta tal cual fuera entregada a los docentes:

1. Como parte del desarrollo de un tema de la asignatura a su cargo: ¿propone la realización de actividades vinculadas con el discurso escrito (lectura y/o escritura) por parte de los estudiantes? (Subraye o resalte una opción)

NUNCA - A VECES- FRECUENTEMENTE - SIEMPRE

2. En caso de haber respondido afirmativamente ¿Cuáles son las actividades que usted propone? (Sea lo más específico posible)

3. ¿Qué espera respecto del desempeño en relación con el discurso escrito por parte de los estudiantes en el contexto de su asignatura? (Sea lo más específico posible)

$1^{\circ}$

Como se observa, la encuesta se organizó en torno de tres preguntas: la $\mathrm{N}^{\circ} 1$ de carácter cerrado y las $\mathrm{N}^{\circ} 2$ y $\mathrm{N}^{\circ} 3$ de carácter abierto.

En la primera, se pretendió indagar la representación imaginaria sobre la propia praxis formativa relativa a la escritura en un plano cuantitativo, y por ello las respuestas preestablecidas en la opción múltiple apuntan a distintos grados de frecuencia al respecto. En 
las dos preguntas de carácter abierto, en virtud de poder sistematizar el análisis, solicitamos estructurar la respuesta en tres instancias diferenciadas (con la posibilidad prevista de que no se completaran necesariamente las tres instancias). La $\mathrm{N}^{\circ} 2$ apunta, al igual que la $\mathrm{N}^{\circ} 1$ pero desde un punto de vista cualitativo (centrado en el "qué" y no en el "cuánto"), al plano de las representaciones respecto de las propias prácticas relativas a la escritura, mientras que la $\mathrm{N}^{\circ} 3$ al de su orientación deóntica, es decir, a lo que los docentes consideran que "hay que" o "se debe" considerar respecto de la enseñanza de la escritura y el desempeño de los estudiantes en relación con ello.

En cuanto al análisis cualitativo de las respuestas a las dos preguntas abiertas, para poder arribar a las principales tendencias en las representaciones docentes sobre el dominio de la escritura, nos centramos en el análisis y clasificación de las operaciones de semiotización-transformación -entendidas como formas constantes en que todo discurso semiotiza el mundo $y$, en virtud de ello, identificables a partir de categorías gramaticales- en tanto indicadores: operaciones de acción, identificación y calificación (Charaudeau, 1995, 1983). Esto es así puesto que, como puede inferirse en virtud del modo en que conceptualizamos supra el orden de las representaciones sobre la escritura estudiadas, el foco está puesto en el carácter necesariamente semiótico-discursivo de las representaciones y en una consideración del discurso en términos de producción social de sentido a partir del cual "el mundo" -o, más particularmente, determinado "aspecto del mundo"- es organizado (y, de este modo, "fundado") semióticamente. Es ello lo que sustenta, en consecuencia, la posibilidad de que formulemos en carácter de indicadores analíticos a las operaciones de transformación postuladas por Charaudeau.

Asimismo, interesa añadir que, mediante estas preguntas de carácter abierto sometidas al análisis cualitativo, se esperaba -en 
virtud de que en ambas se planteó "sea lo más específico posible"que en las respuestas-declaraciones se referenciaran tanto las prácticas u operaciones demandadas al sujeto-estudiante como las entidades u objetos de lectura y escritura asociadas a tales prácticas. Esto es así puesto que, como planteamos en un inicio, partimos de la consideración de que la escritura constituye un dominio de simbolización complejo y multidimensional que involucra -entre otras variables- operaciones (asociadas a sujetos) y objetos.

De esta forma, procuramos específicamente relevar en el análisis, por un lado, la lógica operacional a la que se asocia el dominio de la escritura en el discurso docente, bajo la asunción de que es en función del grado de especificación de los procedimientos asociados a la escritura que se delimita una determinada concepción de sujeto cognoscente y, más específicamente, de "sujeto lector/escritor". Por otro lado, también buscamos analizar el grado de especificidad con que se organiza el dominio de la escritura en función de los objetos y, por tanto, nos centramos en la formulación (o ausencia de formulación) de un metalenguaje en la constitución de las entidades de lectura y escritura en las respuestas-declaraciones, ya sea las que aluden al orden de las prácticas (pregunta $\mathrm{N}^{\circ}$ ) como las que aluden a la dimensión deóntica o expectativas de las prácticas (pregunta $\mathrm{N}^{\circ} 3$ ).

Así pues, como veremos en la próxima sección destinada a la presentación de los resultados, es en virtud de este marco conceptual y de los objetivos que perseguimos que se habilita el relevamiento y clasificación de las operaciones a partir de un examen de determinadas categorías gramaticales tales como "tipos de verbo" o "sustantivos deverbales", para las operaciones de acción, y "sintagma nominal" y "sintagma preposicional" para las operaciones de identificación y calificación, respectivamente.

Por último, resta puntualizar que para el análisis cuantitativo, previsto tanto para las respuestas ante la pregunta cerrada de 
opción múltiple como para las declaraciones-respuestas ante las preguntas abiertas (una vez identificadas y sistematizadas sus constantes tras el análisis cualitativo propuesto), elaboramos una pequeña base de datos con el programa SPSS 17.0 (Statistical Package for the Social Sciences) y posteriormente realizamos estadísticas descriptivas para relevar las frecuencias (absolutas y relativas) de las distintas tendencias. El tratamiento cuantitativo deviene relevante no solo a los fines de establecer una caracterización más exhaustiva de los datos sino también en la medida en que, como plantea Beltrán (2002), a partir de sus resultados -no siempre evidentes en una observación cualitativaes posible realizar inferencias que posibilitan una comprensión más compleja del objeto o fenómeno estudiado.

En las siguientes secciones, nos detendremos en la exposición y discusión de los resultados obtenidos.

\section{Presentación de resultados Análisis cualitativo}

La pregunta $\mathrm{N}^{\circ} 2$ de la encuesta realizada (¿Cuáles son las actividades que usted propone [en relación con el discurso escrito (lectura/escritura)]?) intentó pesquisar en el discurso docente las representaciones que estos sostienen y movilizan sobre su propia praxis formativa relativa a la escritura. Esto es, se trató de una indagación orientada a analizar la manera en que docentes de distintas asignaturas en la escuela secundaria (excluyendo, como dijimos, el área de Lengua) semiotizan el tipo de actividades que promueven en relación con el dominio de la escritura en sus clases. Asimismo, como adelantamos en el apartado anterior, mediante esta pregunta se esperaba que en las respuestas se referenciaran tanto las prácticas u operaciones demandadas al sujeto-estudiante como las entidades u objetos de lectura y escritura asociadas a tales prácticas. 
De hecho, ambas cuestiones han podido ser efectivamente relevadas en el análisis en virtud del tipo de respuestas instanciadas. Justamente, interesa puntualizar que, pese a que no estaba solicitado que se formulara necesariamente de tal forma, casi todas las respuestas a esta pregunta abierta supusieron una formulación a partir de la misma estructura lingüístico-discursiva, lo que no solo reafirma nuestra definición y caracterización del dominio de la escritura formulada supra sino que, además, facilitó el análisis propuesto a partir de la identificación de las operaciones de semiotización: referenciación de la actividad a partir de un sustantivo deverbal (operación de acción mediante nominalización) y planteo de la/s entidad/es involucrada/s en la actividad a partir de una complementación mediante un sintagma preposicional (operaciones de identificación y calificación mediante el sintagma preposicional: "de + sintagma nominal"). Cabe señalar que en los pocos casos en que no se sigue esta estructura se utiliza un infinitivo para el planteo de la actividad y un sintagma nominal para la referencia de las entidades. Asimismo, también se presentan algunos pocos casos -que, como luego veremos, son parte necesariamente del primer valor- en los que no se plantea una complementación del sustantivo deverbal o del verbo en infinitivo mediante sintagma preposicional o nominal (respectivamente) sino una calificación de la acción mediante locuciones adverbiales o adjetivos con valor adverbial.

Teniendo en cuenta lo antedicho, los valores o principales tendencias a las que arribamos son las siguientes:

2. a. Lectura y/o escritura en términos poco o nada específicos

2. b. Lectura y/o escritura como actividad específica y delimitada en cuanto a su objeto

2. c. Operaciones cognitivas o procedimientos vinculados con la lectura y/o escritura

2. d. Otras (no responde / no corresponde) ${ }^{1}$

${ }^{1}$ En esta tendencia incluimos tanto las respuestas no pertinentes en relación con la pregunta efectuada como la ausencia de respuesta en alguno de los tres espacios estipulados para responder. Consideramos relevante su inclusión en la medida en que la ausencia de respuesta 
Así, en primer lugar, en las declaraciones de los docentes respecto de sus prácticas, hallamos una tendencia que denominamos "Lectura y/o escritura en términos poco o nada específicos" (2.a). En este valor se incluyen todas aquellas respuestas cuya representación de la escritura no implica ni moviliza ningún tipo de puntualización semiótico-discursiva sobre el tipo de prácticas y objetos a los que se alude.

Como es de esperarse, se trata de un valor que comprende distintas modulaciones relativas a la "inespecificación". Es decir, en el corpus de respuestas se presenta tanto el empleo de sustantivos deverbales o infinitivos que dan cuenta cuasi tautológicamente de las actividades solicitadas por la pregunta de la encuesta (ej.: "lectura", "escritura", "leer", "escribir") así como también el planteo de actividades que implican una remisión al valor meramente físico-mecánico o incluso mnemónico de la escritura, con alcances estrictamente áulicos (ej.: "transcribir", "anotar", "copia", "respuestas", "confección”); es decir, se trata de prácticas de escritura que adquieren fundamento y sentido pura y exclusivamente en el marco de las actividades de enseñanzaaprendizaje institucionalizadas, en desmedro de la potencialidad epistémica, comunicativa o performativa que la escritura posee por fuera de tal contexto en nuestra sociedad.

Asimismo, resulta interesante destacar que, en el marco de estas respuestas, ambos tipos de predicaciones se formulan acompañadas por complementos mediante sintagmas preposicionales que aluden a entidades sin especificación discursivo-genérica alguna o bien, fundamentalmente -aquí también-, a entidades escolares de escritura, esto es, objetos de escritura cuyo ámbito de circulación e injerencia no trascienden los límites del aquello que supra denominamos campo de la

o su no pertinencia devienen representativas en relación con las representaciones de los docentes en torno de la escritura, justamente, implicando una escasa conciencia respecto de este objeto de enseñanza. 
discursividad escolar (ej.: "de trabajos prácticos", "de respuestas", "de evaluaciones", etc.).

Por otra parte, también inscribimos dentro de esta tendencia a buena parte de los casos analizados en los que directamente se suscita la formulación de actividades en términos "intransitivos", esto es, sin delimitación nominal alguna ("lectura", "lectura colectiva", "lectura silenciosa", "lectura en voz alta", etc.), de modo tal que el grado de inespecificación o vaguedad de la representación es aquí incluso mayor que en los casos anteriores.

Léanse, a continuación, los siguientes ejemplos representativos de la tendencia:

- Lectura en voz alta y relectura individual (encuesta $\left.\mathrm{N}^{\circ} 23\right)^{2}$

- Lectura del tema dado (encuesta $N^{\circ} 12$ )

- Transcribir la idea en la carpeta (encuesta $N^{\circ} 24$ )

- Elaboración de respuestas (encuesta $\mathrm{N}^{\circ} 7$ )

- Confección de trabajos prácticos (encuesta $\mathrm{N}^{\circ} 4$ )

En definitiva, se trata de una tendencia según la cual las prácticas formativas relativas al dominio de la escritura en el imaginario de los docentes encuestados aparecen asociadas a actividades y objetos carentes de especificidad semiótica, comunicativa o epistémica, en la medida en que se formulan operaciones y entidades no delimitadas, intransitivas y carentes de metalenguaje, o bien, con un metalenguaje que no trasciende la funcionalidad escolar-áulica. Consideramos que este tipo de formulaciones involucra una consideración respecto de la escritura

\footnotetext{
${ }^{2}$ Si bien, a los fines de posibilitar el procesamiento en la base de datos organizada para el análisis cuantitativo-estadístico, las Unidades de Análisis (UA) de la investigación corresponden a las 149 declaraciones-respuestas docentes tomadas individualmente, dado que estas fueron extraídas de las 25 encuestas realizadas, para la presentación de los casos y ejemplificación registramos la numeración de las encuestas, justamente, en tanto que fuentes primarias de las UA.
} 
por parte de los docentes encuestados asociada a una configuración del sujeto cognoscente cuyos rasgos agentivos aparecen invisibilizados o puestos en segundo plano. Esto es, en estas declaraciones se presenta un imaginario que, lejos de instituir sujeto agente activo y con objetivos específicos dentro de la cultura escrita, lo que establece es un sujeto cuyo rasgo predominante es el de "alumno" o "estudiante".

La segunda tendencia identificada (2.b) en las respuestas docentes en relación con sus prácticas corresponde a aquella que denominamos "Lectura y/o escritura como actividad específica y delimitada en cuanto a su objeto". Se trata justamente de un subgrupo de declaraciones que formulan y delimitan las prácticas relativas al dominio de la escritura en términos semióticocomunicativos, sobre todo en contraste con la tendencia antes presentada. Léanse los siguientes ejemplos extraídos del corpus:

- Lectura de documentos (encuesta $\mathrm{N}^{\circ} 19$ )

-La lectura de noticias periodísticas de distintos diarios sobre temas dados en clase (encuesta $\mathrm{N}^{\circ} 10$ )

- Confección de presentaciones de power point (encuesta $N^{\circ} 22$ )

- Lectura de teoría escrita (encuesta $\mathrm{N}^{\circ} 9$ )

- Redacción de textos de distinto tipo, en general descripciones y exposiciones de determinados fenómenos de la realidad estudiada (encuesta $\mathrm{N}^{\circ} 11$ )

Como se puede observar, el mayor punto de diferenciación con el primer valor se establece por la manera en que se semiotiza la delimitación de la actividad a partir de los sintagmas preposicionales, y no tanto por la manera en que se semiotiza la actividad en sí. Esto es, en la mayoría de los casos de este subgrupo los sustantivos deverbales o infinitivos mediante los que se remite 
a la actividad tienen un grado de vaguedad análoga al subgrupo anterior -y predomina, una vez más, una suerte de "tautología"-. No obstante, si se observa su complementación mediante la cual se remite a géneros discursivos ("de noticias periodísticas de diversos diarios", "de documentos", "de presentaciones de power point"), o a tipo de textos ("descripciones y exposiciones") o bien a especificaciones más de tipo contenidistas ("de teoría escrita"), es evidente que -aunque sin un único criterio de clasificación-, lejos de presentar las prácticas en términos "intransitivos", delimitadas mediante entidades escolares o sin identificación discursiva, se organizan actividades específicas de lectura y escritura, y tal especificación obedece a criterios semióticocomunicativos en particular.

Consideramos que esto supone una representación notablemente distinta de la correspondiente a 2.a en la medida en que, siguiendo a Schneuwly (2008), cuando los discursos sobre la escritura -en tanto que herramientas semióticas de mediación (Bronckart, 2006; Riestra, 2008)- movilizan un vocabulario específico o metalenguaje, se tornan capaces de objetivar la escritura y, así, eventualmente, habilitan una toma de consciencia en los agentes, tanto en relación con el objeto como respecto del posicionamiento del sujeto en relación con el objeto.

Finalmente, el tercer valor (2.c) hallado en las respuestas a esta pregunta de nuestra encuesta corresponde a lo que denominamos "Operaciones cognitivas o procedimientos vinculados con la lectura y/o escritura”. Esta tendencia -que es, ciertamente, la más heterogénea de las tres-involucra todos aquellos casos en los que, en lugar de responder con una actividad semiótica-comunicativa escrituraria en particular, lo que se inscribe en las declaraciones son prácticas que se vinculan con tal dominio pero presentadas de manera sub especificada.

Véanse, a título de ejemplo, los siguientes ejemplos extraídos del corpus: 
- Búsqueda de información, resumen y comprensión (encuesta $\left.\mathrm{N}^{\circ} 12\right)$

- Reflexión en torno de los conceptos leídos y realización de inferencias al respecto (encuesta $\mathrm{N}^{\circ} 13$ )

- Resumen (encuesta $\mathrm{N}^{\circ} 20$ )

- Selección de ideas principales (encuesta $\mathrm{N}^{\circ} 20$ )

- Buscar el significado de todas las palabras y siglas que aparecen en los textos (encuesta $\mathrm{N}^{\circ} 10$ )

- Análisis del contenido de los textos y resolución de las operaciones de las consignas (encuesta $\mathrm{N}^{\circ} 6$ )

Así, por un lado, hallamos aquí la semiotización -mediante el mismo tipo de estructura lingüístico-discursiva que en las tendencias anteriores- de procedimientos específicos involucrados como parte integrante de prácticas más complejas de lectura y escritura tales como "análisis del contenido de los textos", "búsqueda de información", "resolver ejercicios", "buscar el significado de las palabras", etc. Es decir, se trata de prácticas puntuales que, en una relación de inclusión, son parte integrante de las actividades de lectura y escritura. Por otro lado, hallamos también la semiotización de operaciones cognitivas, que por lo general -aunque no de modo exclusivo- se presentan como asociadas a actividades del dominio de la escritura, tales como "reflexión en torno de los conceptos leídos", "realización de inferencias", "resolución de consignas", etc.

Es decir, se trata de la semiotización de los aspectos más operacionales y epistémicos involucrados en las prácticas de lectura y escritura, por lo que en este aspecto se encuentran en un nivel de complejidad y especificidad mayor que las representaciones de las tendencias anteriores. Justamente, son declaraciones que plantean una constitución de las prácticas de 
escritura asociada a actividades intelectuales, de modo que consideramos que implican una constitución del sujeto al que se dirigen en términos cognoscentes y agentivos.

Asimismo, si atendemos a las operaciones de identificación, como se puede observar en los ejemplos citados, las delimitaciones de las acciones semiotizadas se llevan a cabo a partir de entidades que remiten, en la mayoría de los casos, a categorías semióticodiscursivas ("conceptos"; "palabras"; "textos"; "consignas"), aunque también lo hacen con distintos niveles de especificación y criterios de clasificación como en 2.b.

Pues bien, identificadas las tendencias en las respuestas docentes relativas a la pregunta $\mathrm{N}^{\circ} 2$ de la encuesta, a continuación nos abocaremos al análisis cualitativo de las respuestas correspondientes a la pregunta $\mathrm{N}^{\circ} 3$ ("¿Qué espera respecto del desempeño en relación con el discurso escrito por parte de los estudiantes en el contexto de su asignatura?").

La variable de interés en el discurso e imaginario docente que intentó abordar esta pregunta consistió en la representación sobre el aspecto deóntico relativo a la escritura. Es decir, consiste en una interrogación acerca de las expectativas de los docentes respecto del dominio de la escritura en virtud de sus intervenciones formativas -sobre las que puntualmente se interrogó en la pregunta anterior-, a partir de la premisa de que toda expectativa didáctica está regida por ciertas creencias en relación con lo que se "debe conseguir" como resultado de las prácticas. Consideramos que este tipo de indagación nos permite, por un lado, delimitar con mayor profundidad las representaciones del plano imaginario docente sobre el dominio de la escritura en general -puesto que "decir lo que se espera conseguir" en relación con la escritura siempre encierra una determinada concepción de escritura-, así como también, por otro lado, evaluar si se suscita coherencia entre lo que los docentes "dicen que hacen" - pregunta $\mathrm{N}^{\circ} 2-$ y lo que "plantean como objetivo de lo que hacen" -pregunta $\mathrm{N}^{\circ} 3$ - 
En cuanto al tipo de respuestas a esta pregunta, también se manifiesta - como en la pregunta anterior- una cierta matriz en su organización lingüístico-discursiva: en la mayoría de los casos se presenta una modalidad desiderativa organizada mediante subordinada completiva con verbo en presente del subjuntivo seguida por una complementación en infinitivo que delimita específicamente los alcances y objetivos propuestos. Justamente, es en tales construcciones de infinitivo - que en algunas respuestas se plantean de modo directo, sin el verbo regente en subjuntivoen las que centramos nuestro abordaje analítico, no solo por hallarse en prácticamente todas las respuestas -solo alternadas por algunas con sustantivos deverbales-, sino también $y$ fundamentalmente porque es ahí donde se sintetiza nuestra variable de interés.

Pues bien, desde allí, hallamos tres valores en las respuestas acerca de las expectativas y consideraciones deónticas sobre la escritura, que pueden formularse del siguiente modo:

3. a. Desarrollo de aspectos físico-mecánicos y normativos

3. b. Desarrollo de competencias comunicativas

3. c. Desarrollo de un valor epistémico

3. d. $\operatorname{Otras}^{3}$

A continuación, como hicimos en relación con los valores de la pregunta anterior, explicitamos en qué consiste cada uno de ellos.

El primer valor que identificamos en varias de las respuestas docentes (3.a) corresponde a la declaración de expectativas orientadas por un criterio de "corrección" respecto del dominio de la escritura. Es decir, se trata de la formulación de objetivos vinculados con el desarrollo de aspectos tanto físico-mecánicos como normativos vinculados con las prácticas de lectura y

\footnotetext{
${ }^{3}$ Como hicimos para la clasificación de las respuestas de la pregunta anterior, aquí incluimos tanto las respuestas no pertinentes en relación con la pregunta efectuada como la ausencia de respuesta en alguno de los tres espacios estipulados para responder.
} 
escritura. El eje está puesto, así, en una consideración de la escritura desde sus aspectos más convencionales en tanto que "sistema de notación" (Ferreiro, 2011) y, por tanto, implica reivindicar la adquisición de tales aspectos por parte de los estudiantes como resultado de las prácticas formativas.

Véanse los siguientes ejemplos extraídos del corpus:

- que puedan leer de corrido (encuesta $\mathrm{N}^{\circ} 2$ )

- el uso correcto de la escritura (sin errores ortográficos) (encuesta $\mathrm{N}^{\circ} 7$ )

- que logren una lectura fluida (encuesta $\mathrm{N}^{\circ} 16$ )

- que tengan buena ortografía (encuesta $\mathrm{N}^{\circ}$ )

- que logren transcribir lo aprendido (encuesta $\mathrm{N}^{\circ} 23$ )

Como se desprende de los ejemplos, es fundamentalmente en las operaciones de calificación ("de corrido", "correcto", "buena", "fluida", "sin errores ortográficos") en donde se pone en evidencia de manera más explícita esta primera tendencia

acompañadas y en sintonía, por supuesto, con determinadas operaciones de identificación y acción ("ortografía", "transcribir").

Resulta interesante advertir la inscripción de este valor en algunas de las respuestas docentes en la medida en que se trata de una representación que, en sentido estricto, hasta el momento no habíamos hallado en las declaraciones a la pregunta anterior. Es decir, en ninguna de las declaraciones respecto de lo que los docentes "dicen que hacen" hallamos operaciones orientadas al desarrollo de la normativa ni tampoco semiotizaciones que califiquen las prácticas escriturarias en términos de los pares "bueno" / "malo", "correcto" / "incorrecto", propio de este tipo de representación. Por otro lado, este tipo de representación guiada por el criterio de corrección y normativa se trata de una representación asociada a las prácticas tradicionales de enseñanza (Temporetti, 2006; Cucuzza y Pineau, 2002) que nada tiene que 
ver con la lógica de las actividades de escritura orientadas a operaciones epistémicas y comunicativas como las formuladas (aunque de distinto modo) tanto en 2.b como 2.c. Asimismo, si bien la lógica de la corrección está, como dijimos, por lo general asociada a las prácticas tradicionales de la enseñanza escolar, tampoco es posible decir, en sentido estricto, que se trata de una representación sobre la escritura análoga a la hallada en 2.a., en la medida en que allí no hallamos la modalidad apreciativa polar del par "correcto / incorrecto" que aquí sí está presente.

Por consiguiente, y en virtud del objetivo de tipo evaluativo que nos hemos propuesto, es posible afirmar que aquí ya encontramos un primer elemento de desajuste en las configuraciones semiotizadas de conocimientos de los docentes en relación con la escritura que puede, en buena medida, devenir indicador para comenzar a comprender algunos aspectos de la problemática de la transmisión de la escritura en el contexto de la escuela secundaria al que hicimos referencia en un inicio. No obstante, en función de que, como puntualizamos en la sección precedente, el orden de las representaciones presenta, por definición, una tensión entre homogeneidad y dispersión (Angenot, 2010; Charaudeau, 2012) y sumada la necesidad de atender también al aporte que supone conocer la distribución cuantitativa de las tendencias halladas (que veremos en la próxima sección)-, consideramos que no sería posible ni adecuado por el momento establecer conclusiones unidimensionales ni taxativas al respecto.

En efecto, la segunda tendencia en relación con los aspectos deónticos de la escritura desde el imaginario docente, que denominamos "desarrollo de competencias comunicativas" (3.b), lejos de sostener el criterio de corrección moviliza y reivindica el criterio de "adecuación". Esto es, se trata de todas aquellas respuestas en las que los docentes formulan expectativas y objetivos de escritura vinculados con sus potencialidades específicamente discursivas antes que normativas, por lo que la 
escritura se asocia aquí con las nociones de semiosis, comunicación, comprensión, expresión. Léanse, a título de ejemplo, los siguientes casos:

- espero que desarrollen la capacidad de interpretación (encuesta $\left.\mathrm{N}^{\circ} 20\right)$

- que puedan expresarse con precisión (encuesta N²1)

- que sientan que existe un medio para expresarse, y lograr comprender algo en esa expresión (encuesta $\mathrm{N}^{\circ} 11$ )

- que les permita expresar de manera concreta y simple las ideas que desean transmitir (encuesta $\mathrm{N}^{\circ} 8$ )

- mi objetivo es que sean capaces individualmente, de elaborar una redacción coherente, que permita darse a entender a sus pares, ya sea en una nota de reclamo o simplemente en una carta de intención (encuesta $\mathrm{N}^{\circ} 5$ )

Ahora bien, como se observa en los casos relevados, si bien la constante aquí está dada, como dijimos, por la reivindicación de las potencialidades comunicativas y expresivas de las actividades de escritura -patentizado fundamentalmente en las operaciones de acción planteadas-, es evidente que -con la excepción del último ejemplo citado- en la mayoría no se presenta una semiotización de las entidades de escritura en términos discursivo-genéricos. Esto pareciera ser una suerte de contrasentido (a diferencia de lo que más arriba observamos, aquí el "desajuste" de representaciones se suscita en el interior de las mismas formulaciones) puesto que la consideración comunicativa y el criterio de adecuación implican necesariamente y por definición tal criterio de clasificación: justamente, si no hay género discursivo (Bajtín, 1982) en juego, el criterio de adecuación deviene abstracto o carente de asidero empírico.

Finalmente, planteamos como tercera tendencia en relación con esta pregunta lo que denominamos "desarrollo de un valor epistémico" (3.c). Con esto hacemos referencia específicamente a 
todas aquellas formulaciones de expectativas en las que la lectura y/o escritura se valora en función de su potencialidad para la adquisición de conocimientos. En particular, son formulaciones de objetivos que, al hacer referencia a la injerencia de la escritura como dominio tanto para el desarrollo de conocimientos disciplinares-curriculares como para la ejecución o puesta en acto de operaciones cognitivas en general, movilizan una consideración de la escritura como instancia de mediación epistémica.

Léanse, como ejemplo, los siguientes casos extraídos del corpus:

- Espero, primero que desarrollen un esquema mental que les permita identificar los conceptos de la materia mediante la escritura o la lectura de un texto (encuesta $\mathrm{N}^{\circ} 11$ )

- Que puedan construir su propio conocimiento (encuesta $\mathrm{N}^{\circ} 7$ )

- Aprender a estudiar pensando más y mejor (encuesta $\mathrm{N}^{\circ} 13$ )

- Que puedan resolver eficientemente los ejercicios (encuesta $\mathrm{N}^{\circ} 19$ )

Como se puede inferir, esta representación en torno de las expectativas y objetivos respecto de las actividades de escritura se plantea en estrecha relación con la tendencia 2.c que presentamos supra sobre la representación de las propias prácticas ("operaciones cognitivas o procedimientos vinculados con la lectura y/o escritura") dado que en ambos casos se pone en circulación una noción de escritura entendida como actividad intelectual asociada a la construcción de saberes y a la realización de operaciones particulares. Así pues, a diferencia de lo que observamos anteriormente, aquí se advierte una relación de coherencia entre el plano imaginario de las prácticas y el de su orientación deóntica; en efecto, de ambas tendencias es posible inferir una misma constitución del sujeto al que se dirigen en términos cognoscentes. 
Luego de la descripción de los resultados del análisis cualitativo, en la próxima subsección, procederemos a explicitar los resultados del análisis estadístico, tanto respecto de los valores aquí delimitados como en relación con la primera pregunta cerrada de opción múltiple.

\section{Análisis cuantitativo-estadístico}

En relación con la pregunta $\mathrm{N}^{\circ} 1$ ("Como parte del desarrollo de un tema de la asignatura a su cargo: ¿propone la realización de actividades vinculadas con el discurso escrito -lectura y/o escritura- por parte de los estudiantes? Subraye o resalte una opción: Nunca - A veces - Frecuentemente - Siempre"), orientada a indagar las representaciones sobre la propia praxis acerca de la escritura en términos de frecuencia cuantitativa, las respuestas docentes se distribuyeron del siguiente modo:

Tabla 1

Frecuencia en actividades con discurso escrito

\begin{tabular}{lrrr}
\hline & Frequency & Percent & Valid Percent \\
\hline Nunca & 0 & 0 & 0 \\
A veces & 3 & 12,0 & 12,0 \\
Frecuentemente & 11 & 44,0 & 44,0 \\
Siempre & 11 & 44,0 & 44,0 \\
Total & 70 & 100,0 & 100,0 \\
\hline
\end{tabular}

Los datos son contundentes: la gran mayoría (88\%) -dividida en partes iguales entre los que responden "siempre" y "frecuentemente"- considera que en sus actividades formativas la escritura tiene un lugar relevante, y absolutamente ningún docente responde que "nunca" realiza actividades de escritura. 
Este resultado nos interesa en la medida en que mostraría el arraigo de determinadas representaciones que históricamente han dominado el contexto del campo de la discursividad escolar. Esto es, en particular, pese a que en las últimas prescripciones curriculares vigentes a nivel provincial (Ministerio de Educación de la Provincia de Santa Fe, 2014) se puede leer de modo bastante explícito una reivindicación de prácticas formativas centradas en otros lenguajes y dominios más allá de la centralidad de la palabra escrita, con un foco puesto en lo experiencial, la interacción oral, lo audiovisual y las nuevas tecnologías en general (Condito, 2018), si nos centramos en los números de esta muestra, se pone de manifiesto que en el plano imaginario o en los mundos representados de los docentes la escritura continúa siendo un pilar o valor decisivo y constitutivo de la educación secundaria, tal como establece el contrato histórico-fundacional del nivel (Cucuzza y Pineau, 2002).

En cuanto a la pregunta $\mathrm{N}^{\circ} 2$, vinculada con el modo en que los docentes se representan cualitativamente sus actividades formativas, tenemos los siguientes resultados:

Tabla 2

Tipo de actividad en relación con discurso escrito

\begin{tabular}{lrrr}
\hline & Frequency & Percent & Valid Percent \\
\hline 2.1: actividad no especificada & 31 & 44,3 & 44,3 \\
2.2: actividad especificada & 15 & 21,4 & 21,4 \\
2.3: operación cognitiva & 19 & 27,1 & 27,1 \\
no pertinente & 5 & 7,1 & 7,1 \\
Total & 70 & 100,0 & 100,0 \\
\hline
\end{tabular}

Como se observa, proporcionalmente, la gran mayoría de los docentes encuestados (44.3\%) manifiesta en su respuesta una 
tendencia a plantear actividades "no específicas" - -sin delimitación en cuanto a la actividad y en cuanto al objeto, o con una delimitación de tipo escolar-, y el resto se divide entre quienes se centran en los aspectos operacionales-epistémicos (27.1\%) y, en una menor proporción, entre quienes movilizan una representación de la escritura a partir de un metalenguaje específico $(21.4 \%)$.

De estos datos es posible deducir de modo preliminar -sobre todo si advertimos que solo 15 de 70 respuestas se ubican en el polo de la "especificidad"-, una escasa conciencia por parte de estos docentes respecto de las potencialidades de sus mediaciones formativas para la constitución de las prácticas de lectura y escritura en términos de praxis significativa en sí misma, capaz de transcender los límites de la funcionalidad escolar - es decir, capaz de ser algo más que un mero "medio" para enseñar y aprender o para organizar las rutinas áulicas-. Ello puede leerse, asimismo, en relación con los resultados estadísticos anteriores: si bien pareciera haber entre los docentes un acuerdo generalizado en relación con el necesario protagonismo de la lectura y la escritura en las prácticas formativas en el nivel secundario, se vislumbra siguiendo el planteo de Navarro y Revel Chion (2013) - una escasa conciencia o conocimiento respecto de su configuración o transposición en tanto que objeto de enseñanza.

Por su parte, las frecuencias asociadas con la pregunta $\mathrm{N}^{\circ} 3-$ orientada a indagar el aspecto deóntico relativo a las mediaciones formativas sobre la escritura- presentan la siguiente distribución: 
Tabla 3

Tipo de expectativas en relación con discurso escrito

\begin{tabular}{lrrr}
\hline & Frequency & Percent & Valid Percent \\
\hline 3.1: corrección & 8 & 14,8 & 14,8 \\
3.2: adecuación & 29 & 53,7 & 53,7 \\
3.3: valor epistémico & 14 & 25,9 & 25,9 \\
no pertinente & 3 & 5,6 & 5,6 \\
Total & 54 & 100,0 & 100,0 \\
\hline
\end{tabular}

En esta variable las frecuencias de cada tipo de respuesta son aún más contundentes: más de la mitad (53.7\%) se ubican en el criterio de adecuación, seguido por aquellas que se centran en el valor epistémico $(25.9 \%)$, y finalmente se presenta una menor cantidad que se inscribe dentro del criterio de corrección (14.8\%).

Si comparamos estos resultados con los anteriores (plano imaginario respecto de las propias prácticas en términos cualitativos), sobresale el hecho de que no se presenta demasiada coherencia en las representaciones de los docentes en relación con las actividades de escritura (lo que "dicen que hacen") y los objetivos a los que dicen orientarse (lo que dicen acerca del "por qué y para qué" lo hacen). Esto es, mientras que la tendencia predominante en relación con las prácticas formativas, como dijimos, es la de la "no especificación" o la "intransitividad", la predominante respecto de las expectativas es la de la adecuación, lo que en sí mismo y por definición encierra una consideración de la escritura en términos de actividad social, por lo que no condice con actividades en las que no se ponen en juego los valores comunicativos, expresivos y semiótico-discursivos de la escritura. 


\section{Conclusiones y proyecciones del análisis}

En las páginas precedentes realizamos un principio de análisis de determinadas declaraciones y respuestas que forman parte del discurso docente, enmarcado en el campo de la praxis y discursividad escolar. Partimos de la premisa de que este espacio discursivo detenta un sistema de representaciones (Charaudeau, 2012, 2009, 1983; Bronckart, 2004, 2007) que contextualizan y regulan las actividades formativas donde se materializa la transposición didáctica del objeto de enseñanza -en particular, en este caso, del objeto escritura-, y en ello radica la importancia de su exploración y sistematización.

Para sintetizar, la principal tendencia que atraviesa el corpus abordado reside en la representación de la escritura como dominio inespecífico en términos semiótico-discursivos -sobre todo en lo que concierne a sus productos u objetos pero también en relación con las prácticas- y en buena medida asociado con la funcionalidad escolar.

Asimismo, también observamos a raíz de los datos y su distribución, una escasa correlación entre las prácticas formativas declaradas y las expectativas u objetivos formulados en relación con el desarrollo del dominio de la escritura. Ello operaría, en efecto, como un primer indicio respecto de la poca conciencia por parte de los docentes encuestados en lo que concierne a sus intervenciones didácticas asociadas a la escritura.

Consideramos que estos resultados ameritan ser sometidos a examen y problematización puesto que dan cuenta de un desajuste notorio -en el plano del discurso docente- entre praxis y expectativas relativas a la escritura y, en consecuencia, merecen ser sometidos a futuras indagaciones centradas, no ya en el análisis del plano de los "mundos representados" o imaginario como el que aquí propusimos, sino en el de las prácticas e interacciones formativas empíricas llevadas a cabo en las aulas. 
Esto es, aunque de los resultados analíticos se desprende la presencia de una suerte de consenso respecto del lugar jerárquico que la escritura asume en las prácticas formativas, si consideramos la escasa inscripción de metalenguaje específico y atendemos a los problemas de coherencia interna y desajustes mencionados, cabe conjeturar que estamos ante un escenario que demanda nuevas indagaciones $y$, fundamentalmente, intervenciones específicas en la formación docente orientadas a la concientización acerca del dominio de la escritura, sus variables constitutivas y sus potencialidades epistémicas, comunicativas y performativas, de modo tal que se pueda trascender la lógica "inmanente" e "intransitiva" de la escritura dentro de las aulas.

Por otra parte, también interesa señalar que el predominio de una tendencia (la escritura como dominio inespecífico en términos discursivos y asociado con la inmanencia y funcionalidad escolar) no niega su coexistencia con otras representaciones que, ciertamente, también aparecieron como parte de los resultados de la indagación, aunque en menor proporción -como, por ejemplo, la representación de la escritura como instancia que moviliza operaciones cognitivas, o la escritura como conjunto de técnicas asociadas a prescripciones normativas-. Esto es así puesto que la hegemonía (Angenot, 2010; Bourdieu, 2001) de un tipo de concepción sobre la escritura no inhabilita ni impide la existencia y circulación de otro tipo de representaciones, muchas veces opuestas o enfrentadas. Justamente, sostenemos que hay, en efecto, -como hemos intentado mostrar- determinados valores, modulaciones y tendencias dominantes en la consideración del objeto escritura que se materializan en las intervenciones discursivas analizadas; pero ello también da lugar a voces disidentes, contradictorias o heterogéneas que conjuntamente conforman y delimitan la complejidad de un campo (Bourdieu, 2002). 
Para concluir, consideramos que en virtud del conocimiento respecto de las representaciones que sostenemos como docentes acerca de nuestras actividades formativas es posible comenzar a interrogar el proceso de conformación de los objetos de enseñanza a los fines de, eventualmente, poder elaborar proyectos acordes de intervención didáctica destinados a la mejora de la praxis. Dicho de otro modo, estimamos que es a partir de la toma de consciencia respecto de la estructuración y sistemas de creencias o imaginarios que regulan nuestras prácticas que podemos comenzar a pensar en posibles maneras de mejorarlas, por lo que este tipo de análisis puede resultar de utilidad para establecer parámetros capaces de orientarnos en nuestras propias intervenciones docentes, motivadas, siempre, por la necesidad de intervenir y de transformar aquello que necesita ser transformado. 


\section{Referencias bibliográficas}

Angenot, M. (2010). El discurso social. Los límites de lo pensable y lo decible. Buenos Aires: Siglo XXI.

Bajtín, M. (1982). Estética de la creación verbal. México DF, México: Siglo XXI.

Beltrán, C. (2012). Introducción al análisis estadístico en la investigación: con aplicaciones en distintas disciplinas. Rosario: Juglaría.

Bourdieu, P. (2001). ¿Qué significa hablar? Economía de los intercambios lingüísticos. Madrid: Akal.

(2002). Campo de poder, campo intelectual. Itinerario de un concepto. Buenos Aires: Montressor.

(2015). La nobleza de estado. Educación de élite y espíritu de cuerpo. Primera parte. Buenos Aires: Siglo XXI.

Bronckart, J-P. (2004). Actividad verbal, textos y discursos. Madrid: Fundación infancia y aprendizaje.

(2007). Desarrollo del lenguaje y didáctica de las lenguas. Buenos aires: Miño y Dávila.

Bruner, J. (2004). Realidad mental y mundos posibles. Barcelona: Gedisa.

(2009). Actos de Significados. Madrid, España: Alianza.

Calvet, L. J. (2008). Historia de la escritura. De Mesopotamia hasta nuestros días. Buenos Aires: Paidós.

Cárdenas, V. (2013). Sociedad, lenguaje y alfabetización. Acerca del error y del cambio lingüístico. En AA. VV. Hablar y escribir en la Universidad. Salta: EDUNSa.

Cardona, G. (1999). Antropología de la escritura. Barcelona: Gedisa.

Catach, N. (Comp.) (1996). Hacia una teoría de la lengua escrita. Barcelona: Gedisa. 
Cavallo, G. y Chartier, R. (Dirs.) (2004). Historia de la lectura en el mundo Occidental. Madrid: Taurus.

Charaudeau, P. (1983). Langage et discours. Eléments de sémiolinguistique (théorie et pratique). Paris: Hachette.

(1995). Une analyse sémiolinguistique du discours. En Langages $\mathrm{n}^{\circ} 117$. Recuperado de http://www.patrickcharaudeau.com/Une-analyse-semiolinguistique-du,64.html.

(2009). Análisis del discurso en interdisciplinariedad en las ciencias humanas y sociales. En Puig L. (Ed.), El discurso y sus espejos. Universidad Nacional Autónoma de México.

(2012). Entre la pragmática y las teorías de la enunciación. Entrevista a Patrick Charaudeau. En Lordoño Zapata, O. (2012). Poliedros discursivos. Miradas a los estudios del discurso. Villa María: Eduvim.

Chartier, A. M. (2005). Enseñar a leer y escribir. Una aproximación histórica. México DF: Fondo de Cultura Económica.

Chevallard, Y. (1985). La transposition didactique. Du savoir savant au savoir enseigné. Grenoble: la pensée sauvage.

Condito, V. (2014). La relación lectura - escritura: una configuración histórica. Apuntes para un análisis. Saga. Revista de Letras $\mathrm{N}^{\circ}$, , de la Facultad de Humanidades y Artes de la Universidad Nacional de Rosario, pp. 234-268.

(2016). "Representaciones sobre la escritura en las consignas. Hacia una primera aproximación en el análisis de datos en una Escuela Media de Rosario". Nothstein, S., Pereira, M. C., Valente, E. (Ed.). Lectura y escritura: prácticas, instituciones y actores. Bahía Blanca: Ediuns - Sociedad Argentina de Lingüística, pp. 261-283.

(2018). La escritura en las mediaciones formativas escolares. Representaciones sobre la escritura en consignas de $1^{\circ}$ y $5^{\circ}$ año de la Escuela Media (Tesis doctoral). Facultad de Humanidades y Artes. Universidad Nacional de Rosario.

Cucuzza, H. (Dir.) y Pineau, P. (Coodir.) (2002). Para una historia de la enseñanza de la lectura y la escritura en Argentina. Del 
catecismo colonial a La Razón de Mi Vida. Buenos Aires: Miño y Dávila.

Delicia Martínez, D. (2016). Prólogo. En Muse, C. (Ed.), Lectura y escritura en el nivel medio (Vol. 2 de Colección Cátedra UNESCO, Lectura y escritura: continuidades, rupturas y reconstrucciones). Córdoba: UNC editora.

Desinano, N. y Solana, Z. (Coord.) (2015). Lectura y escritura: Cuadernos de Investigación 1. Rosario: FHUMyAR Ediciones.

Falchini, A. (2006). Pensar con palabras. La escritura: un objeto cultural complejo. En AAVV, Lengua y Literatura. Prácticas de enseñanza. Perspectivas y propuestas, Santa Fe: UNL ed.

Ferreiro, E. (2011). Cultura escrita y educación. México DF: Fondo de Cultura Económica.

Freytes, M. F. y Fernández, M. V. (2016). Prólogo. En Muse, C. (Ed.), Lectura y escritura en el nivel superior (Vol. 3 de Colección Cátedra UNESCO, Lectura y escritura: continuidades, rupturas y reconstrucciones). Córdoba: UNC editora.

Ministerio de Educación de la Provincia de Santa Fe (2014). Diseño Curricular de Educación Secundaria Orientada. Recuperado de https://www.santafe.gov.ar/index.php/educacion/content/dow nload/218364/1135170/file/Anexo\%20III\%20Resol\%20263014.pdf.

Navarro, F. y Revel Chion, A. (2013). Escribir para aprender. Disciplinas y escritura en la escuela secundaria. Buenos Aires: Paidós.

Olson, D. (1994). El mundo sobre el papel. Barcelona: Gedisa.

Olson, D. y Torrance, N. (Comp.) (1998). Cultura escrita y oralidad. Barcelona: Gedisa.

Pérez, L. y Rogieri, P. (Dirs.) (2016). Retóricas del decir. Lenguaje, verdad y creencia en la escritura académica. Rosario: FHUMyAR ediciones. $2^{\mathrm{a}}$ edición revisada.

Petrucci, A. (1999). Alfabetismo, escritura y sociedad. Barcelona: Gedisa. 
Riestra, D. (2008). Las consignas de enseñanza de la lengua. Un análisis desde el interaccionismo sociodiscursivo. Buenos Aires: Miño y Dávila.

(2010). "El trabajo docente en la enseñanza de la lengua: los textos y el análisis entre los géneros y los tipos de discurso". Castel, V. y Cubo de Severino, L., (Eds.) (2010). La renovación de la palabra en el bicentenario de la Argentina. Los colores de la mirada lingüística. Mendoza: Editorial FFyL, UNCuyo.

Schneuwly, B. (2008). Vygotski, l'école et l'écriture. Genève: Université de Genève, Faculté de psychologie et des sciences de l'éducation.

Tedesco, J. C. (1993). Educación y sociedad en la Argentina (18801945). Buenos Aires: Solar.

Temporetti, F. (2006). Prácticas educativas: entre lo individual y lo sociocultural. Breve ensayo sobre los conocimientos psicológicos en la enseñanza. En Itinerarios Educativos, la revista del INDI, FHU, Año 1, No 1, pp. 89-102.

Fecha de recepción: 26/03/2019

Fecha de aprobación: 10/o6/2019 